\title{
Focal Fibrous Hyperplasia: A Rare Case Report and Literature Review
}

\author{
Akhil Jose EJ*, Prashant Babaji and Shashibushan KK \\ Department of Pedodontics and Preventive dentistry, Sharavathi Dental College and Hospital, India
}

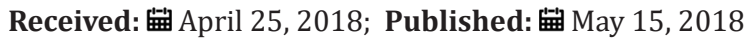

*Corresponding author: Akhil Jose EJ, Department of Pedodontics and Preventive dentistry, Sharavathi Dental College and Hospital, Shimoga, Karnataka, India

\begin{abstract}
Intra-oral fibrous type soft tissue overgrowth is common in oral cavity. It can be either benign or neoplastic lesions. Nonneoplastic type of reactive lesion seen in the oral cavity is caused by the chronic irritation. Focal fibrous hyperplasia is one such lesion which is seen in the line of occlusion. Focal fibrous hyperplasia is also known as irritational fibroma or traumatic fibroma. Proper oral hygiene, removal of etiologic factors and conservative excision will prevent the focal fibrous hyperplasia and its recurrence. Proper diagnosis, management and follow up should be done in these cases.
\end{abstract}

Keywords: Benign tumour, Focal fibrous hyperplasia, Irritation fibroma

Abbreviations: PG: Pyogenic Granuloma; PGCG: Peripheral Giant-Cell Granuloma; POF: Peripheral Ossifying Fibroma

\section{Introduction}

A large number of soft tissue enlargements can be seen in the oral cavity. Most of these pathological over growth are reactive hyperplastic lesions seen in response to chronic inflammation caused by chronic irritation [1]. Focal fibrous hyperplasia or Irritational fibroma or Traumatic fibroma is one of the most common epithelial benign tumours of the oral cavity $[2,3]$. These localized progressive, proliferation of the oral mucosa are seen in response to injury or local irritation from dental plaque, calculus, sharp edge of grossly decayed teeth, fractured teeth, trapped food particles, ill-fitting dental/oral appliances, food impaction and iatrogenic factors (extended flanges of the denture and over extended dental restorations)[1,3]. These non-neoplastic lesions seen on the buccal mucosa along the line of occlusion (subjected to masticatory trauma) causes esthetic and functional problems [2,4,5]. These sessile lesions are seen on the gingiva, lower lip, tongue, hard palate and edentulous alveolar ridges [2,4]. Focal fibrous hyperplasia affect people of all ages, but females are twice more likely to develop focal fibrous hyperplasia $[2,3]$. This is because the female hormones may cause an increased production and accumulation of collagen by fibroblast in the presence of chronic injury [2]. Focal fibrous hyperplasia is more common in maxillary region and seen more often in the incisor-cuspid region [6]. It usually presents as a asymptomatic smooth surface soft nodule of yellowish- white colour. Predilection of focal fibrous hyperplasia in adult is due to the mechanical trauma caused by wearing dentures, lower lip biting habit, chronic cheek-biting and biting objects [2]. The lesion gradually increases in size with no history of bleeding and pain. Similar lesions like Pyogenic Granuloma (PG), Peripheral Giant-Cell Granuloma (PGCG) and Peripheral Ossifying Fibroma (POF) may also arise in the oral cavity as a result of irritation due to plaque microorganism and other local irritations which are ruled out from focal fibrous hyperplasia by histologic analysis [2,7]. These lesions are mainly seen in the interdental papilla region. Focal fibrous hyperplasia are asymptomatic painless lesion with variable surface appearance which may be ulcerated / non- ulcerated [1]. It may occur again if etiology is not eliminated [3].

\section{Case History}

A 12 year old female patient came to the Department of Pedodontics with a chief complaint of a growth in the buccal mucosa of the mouth 3 days before. There is no associated pain or swelling from this growth. Intra-oral examination revealed a localized, well defined soft nodule of $2 \mathrm{~cm} \mathrm{X} 1 \mathrm{~cm}$ in diameter with white colour serrated surface seen on the right buccal mucosa area near the occlusal line of the canine region (Figure 1). No 
other associated signs or symptoms were detected. There was no associated history of trauma and pain. Medical and family history was non-contributory. There were no signs of any syndrome. Blood investigations were done and the reports were well within normal limits. On palpation the growth was nodular, movable, non-compressible, firm, fibrous and non-tender with no signs of any discharge. An intra-oral full mouth scaling was done to remove local irritants. Blood counts were within the normal limits. On the basis of clinical findings, provisional diagnosis of fibrous epithelial hyperplasia was given. Treatment plan for focal fibrous hyperplasia was complete surgical excision. Excisional biopsy was planned and the patient consent was taken for the same. After the infiltration of the anaesthesia far from the lesion, it was holded with a forceps for convenient handling. Lesion was excised using a surgical scalpel blade (Figure 2). No suturing was required (Figure 3) and analgesics were prescribed post-operatively. The resection specimen (Figure 4) was sent to the anatomic-pathology lab for histological examination which confirmed the diagnosis of a fibrous hyperplasia. The wound healing was uneventfully and the patient presented with no post-operative complications (Figure 5).

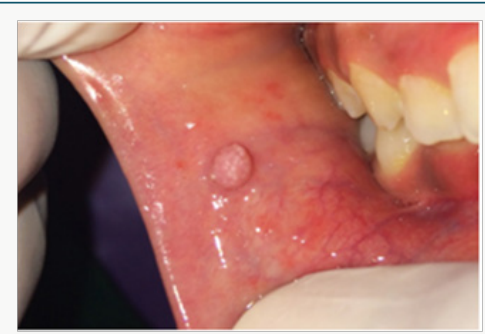

Figure 1: Intra-Oral Photograph Showing Focal Fibrous Hyperplasia in Buccal Mucosa in Line of Occlusion.

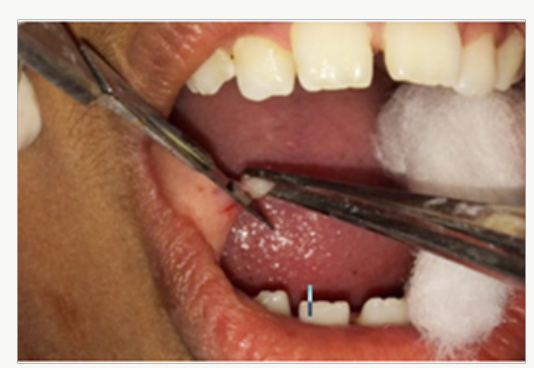

Figure 2: Surgical Removal of Focal Fibrous Hyperplasia Using Scalpel.

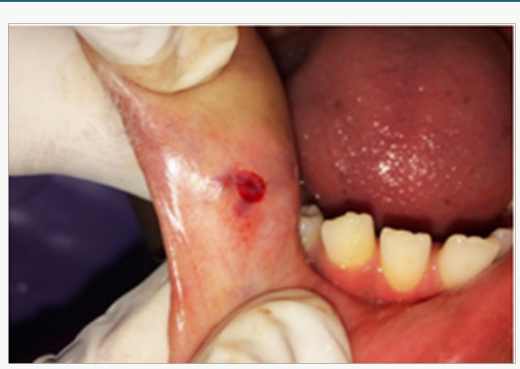

Figure 3: Buccal Mucosa after Removal of Focal Fibrous Hyperplasia.

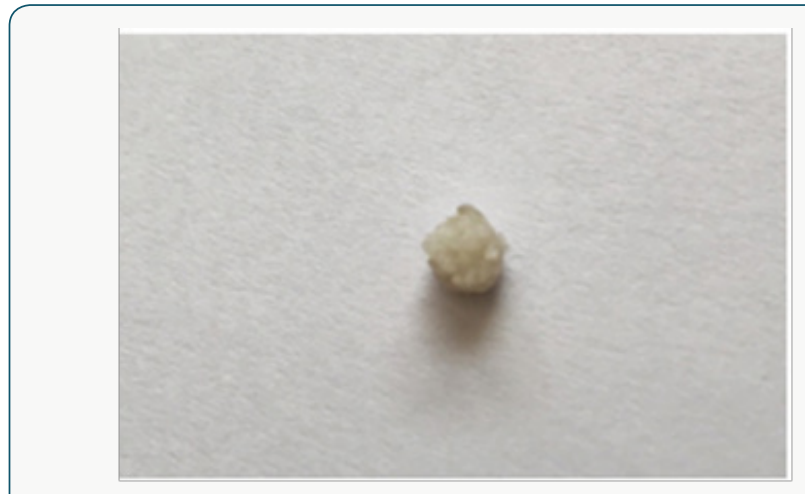

Figure 4: Surgically Removed Focal Fibrous Hyperplasia.

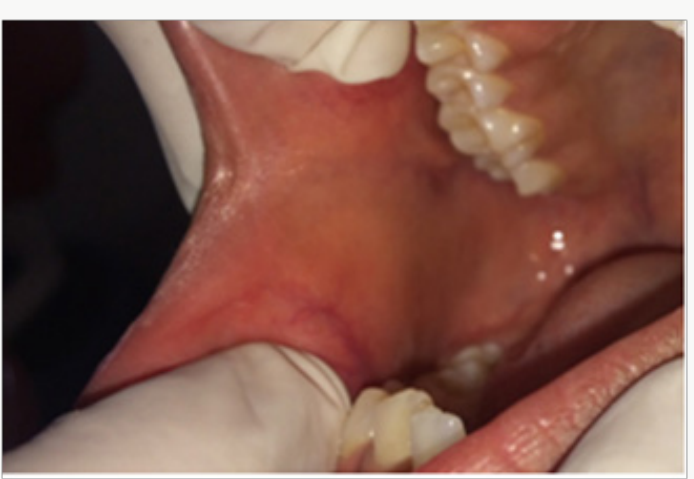

Figure 5: Uneventfully Healed Surgical Site After 2 Weeks.

\section{Discussion}

High frequency of tissue injuries or chronic irritation in the oral cavity cause reactive lesion. Fibroma occurs as a result of a chronic repair process that includes granulation tissue and scar formation resulting in a sub mucosal fibrous mass [3]. Cheek biting is one of the main reason for the occurrence of fibroma along the occlusal plane. These overgrowth lesions caused by low grade stimulus are managed by efficient plaque control, removal of etiological factors and excision of the over growth [8]. Very rarely recurrences of focal fibrous hyperplasia have been reported after excision [3]. Although recurrence of the lesion is low, the follow up is must [8]. The scalpel, lasers, and conventional electro surgery unit are the instruments of choice for soft tissue surgery. Because of their ease of use, accuracy, and minimal damage to the surrounding tissue scalpels are used commonly. Due to the limitation of difficulty in achieving haemostasis in scalpel surgery, new advances like LASER and Electro surgery unit came into the field of dentistry. They provide a greater precision, a relatively bloodless surgical and postsurgical course, sterilization of the surgical area, minimal swelling and scarring, coagulation, vaporization, minimal or no suturing, and much less or no postsurgical pain. Diode lasers are also used for soft tissue excision [5]. Laser treatment produces less scar tissue contraction, maintains the elastic tissue properties, avoids the use of post-operative medications and accelerates the healing process [2]. A focal fibrous hyperplasia does not pose a risk of malignancy [3]. 


\section{Conclusion}

Majority of the localized intraoral gingival lesions have slowly progressing growth. As these lesions are mostly asymptomatic, patient used to take treatment once the lesion becomes visible and if they interfere in mastication, speech or if any unesthetic disfiguring in the anterior region. Patient compliance and motivation along with proper diagnosis, prevention, management and treatment of these lesions are of the utmost importance. Treatment involves removal of local irritation, conservative complete surgical excision of the lesion and regular follow-up to prevent the recurrence.

\section{References}

1. Binita G, Jigar D, Abraham J, Disha B (2016) Reactive Lesions of Oral Cavity. Natl J Integr Res Med 7(4): 154-157.
2. Thiago de Santana Santos T, Martins Filho PS, Piva MR, de Souza Andrade ES (2014) Focal fibrous hyperplasia: A review of 193 cases. J Oral Maxillofac Pathol 18(1): 86-89.

3. Ayekinam K, Karima EH, Wafaa EW (2017) Surgical removal of a focal fibrous hyperplasia: Two case reports. Int J Appl Dent Sci 3(2): 215-217.

4. Astekar M, Gupta S, Soumya G (2011) Focal Fibrous Hyperplasia: Report of two Cases. Int J Dent Clin 3(1): 111-112.

5. Pardeshi KV, Mirchandani NM, Agrawal AA, Kale TM (2016) Fibrous hyperplasia: Two case reports. Dent Lasers 10(1): 23-27.

6. Arora SA, Chhina S, Goel A, Mishra S, Kazimm J, et al. (2016) Irritational Fibroma: A Case Report. Int J Oral Health Med Res 2(5): 78-80.

7. Kolte A, Kolte R, Shrirao T (2010) Focal fibrous overgrowths: A case series and review of literature. Contemp Clin Dent 1(4): 271-274.

8. Shah SJ, Datar UV, Pharne P, Kanitkar SS, Kamat M (2017) Focal Reactive Overgrowths: Case Series with Review of Literature. J Clin Diagn Res 11(12): 1-4.
(C) (1) This work is licensed under Creative

To Submit Your Article Click Here: Submit Article

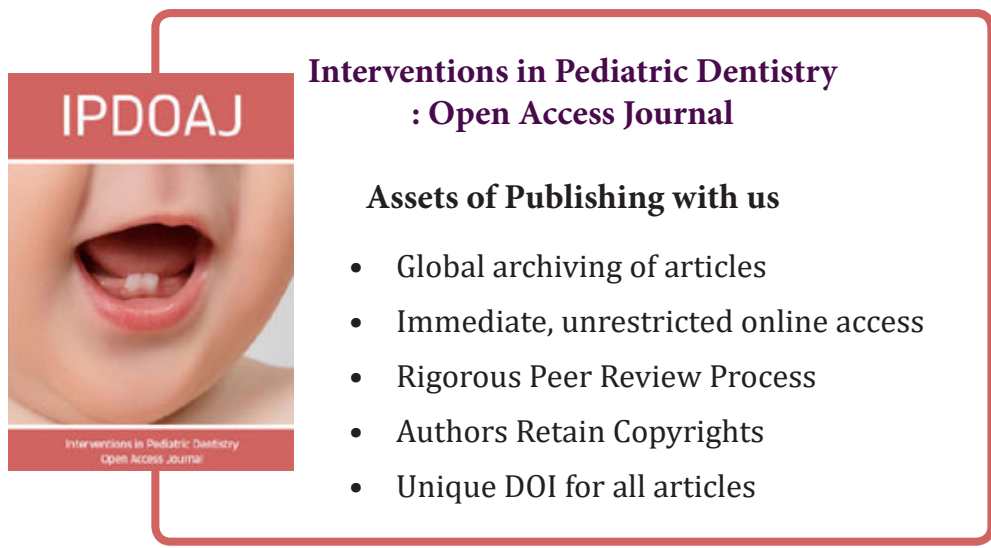

